\title{
Utility of F-18 Fluorothymidine PET Over F-18 Fluorodeoxyglucose PET/CT in Detection of a Recurrent Glioma
}

Raghava Kashyap, Manoj Kumar Tiwari, Raja Senthil, Anish Bhattacharya, Vivek Gupta, Bhagwant Rai Mittal

\begin{abstract}
Differentiation of postsurgical or postradiation necrosis from tumor recurrence is a clinical dilemma. Magnetic resonance imaging does not always perform well in this setting. Several nuclear medicine techniques are available that offer a solution in such a clinical situation. We report a case where F-18 flurodeoxyglucose (FDG) and F-18 fluorothymidine (FLT) PET/ CT were used to identify recurrence of a glioma.
\end{abstract}

Keywords: Glioma, F-18 FDG, F-18 FLT, PET/CT.

How to cite this article: Kashyap R, Tiwari MK, Senthil $\mathrm{R}$ Bhattacharya A, Gupta V, Mittal BR. Utility of F-18 Fluorothymidine PET Over F-18 Fluorodeoxyglucose PET/CT in Detection of a Recurrent Glioma. J Postgrad Med Edu Res 2012;46(4):202-203.

Source of support: Nil

Conflict of interest: None declared

\section{INTRODUCTION}

Differentiating recurrent gliomas in a postoperative and postradiotherapy setting remains a challenge. Although magnetic resonance imaging (MRI) has superior sensitivity in lesion detection, its specificity is reported to be as low as 27\%. ${ }^{1}$ Several nuclear medicine techniques are available as a solution to this problem, e.g. Tc99m labeled glucoheptonate (GHA) or methoxyisobutylisonitrile (MIBI) SPECT, F-18 flurodeoxyglucose (FDG) positron emission tomography/computed tomography (PET/CT), F-18 fluorothymidine (FLT), F-18 F-DOPA and C-11 Methionine PET/CT. ${ }^{2-5}$

\section{CASE REPORT}

A 25-year-old female patient with a right temporal glioma underwent craniotomy and excision of the tumor in December 2010. Histopathology revealed a grade II astrocytoma. She presented 6 months later with vertigo, nausea and disorientation. Recurrence of tumor was suspected. The patient underwent F-18 FDG and F-18 FLT PET/CT studies. The FDG study revealed intense tracer uptake (SUVmax, 15.0) in the right temporal pole (Fig. 1A), and the same focus was also demonstrated on the FLT PET/ CT (Fig. 1B). However, apart from this lesion, the FDG PET/CT showed another focus of abnormal uptake in the periventricular region surrounding the anterior horn of the lateral ventricle on the left side (Fig. 1C) while the FLT $\mathrm{PET} / \mathrm{CT}$ demonstrated increased tracer uptake in the periventricular regions on the lateral ventricles on both sides (Fig. 1D). MRI showed a T2 hyperintense lesion in the right temporal lobe corresponding to the FDG avid focus (Figs 1E and F). Contrast enhancing lesions were seen in bilateral periventricular-subependymal region and also involving the corpus callosum (Figs $1 \mathrm{G}$ and $\mathrm{H}$ ). On the basis of the imaging findings, diagnosis of tumor recurrence with CSF spread was considered and the patient was scheduled for chemotherapy.

\section{DISCUSSION}

Although high-grade tumors show intense FDG uptake, the physiological uptake in the brain may interfere with accurate interpretation. Tracers like GHA, FLT, dihydroxyphenylalanine (DOPA) and methionine ${ }^{2-5}$ have an advantage in this respect as there is no physiological uptake of these tracers in the brain tissue. PET images have better resolution than SPECT images. FLT can therefore play a significant role in the detection of recurrences of gliomas. Several studies have reported the utility of F-18 FLT in predicting the grade of gliomas. ${ }^{3-8}$ Yamamoto et al in their study demonstrated significant correlation between metabolic volumes of recurrent glioblastoma multiforme and the volumes defined on MRI. ${ }^{9}$ FLT was also better than methionine in tumor grading. ${ }^{5}$ The uptake of this tracer in lesions is less than that of FDG. However, due to negligible uptake in the normal brain parenchyma, FLT provides a high tumor to background contrast that can help in detection of lesions. The uptake of FLT in low-grade gliomas is expected to be low. ${ }^{3}$ In the present case (a grade II astrocytoma), though the FDG uptake was very high (SUVmax, 15.0) but FLT showed definite abnormal uptake and identified the exact distribution of the recurrent lesions.

\section{REFERENCES}

1. Santra A, Kumar R, Sharma P, Bal C, Kumar A, Julka PK, et al. F-18 FDG PET/CT in patients with recurrent glioma: Comparison with contrast enhanced MRI. Eur J Radiol 2011 Feb 23.

2. Schillaci O, Filippi L, Manni C, Santoni R. Single-photon emission computed tomography: Computed Tomography in brain tumors. Semin Nucl Med 2007;37:34-47.

3. Tripathi M, Sharma R, D’Souza M, Jaimini A, Panwar P, Varshney R, et al. Comparative evaluation of F-18 FDOPA, F-18 FDG, and F-18 FLT-PET/CT for metabolic imaging of low grade gliomas. Clin Nucl Med 2009;34:878-83. 


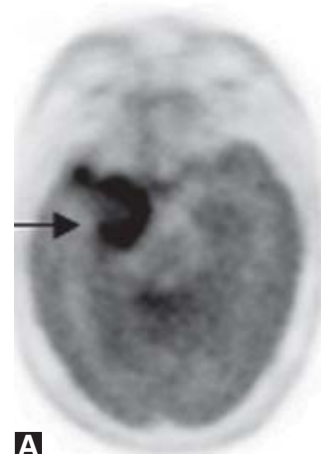

A

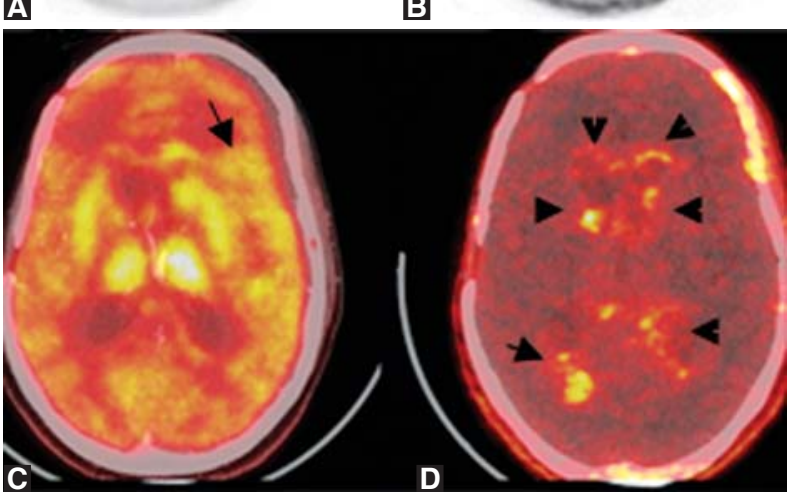

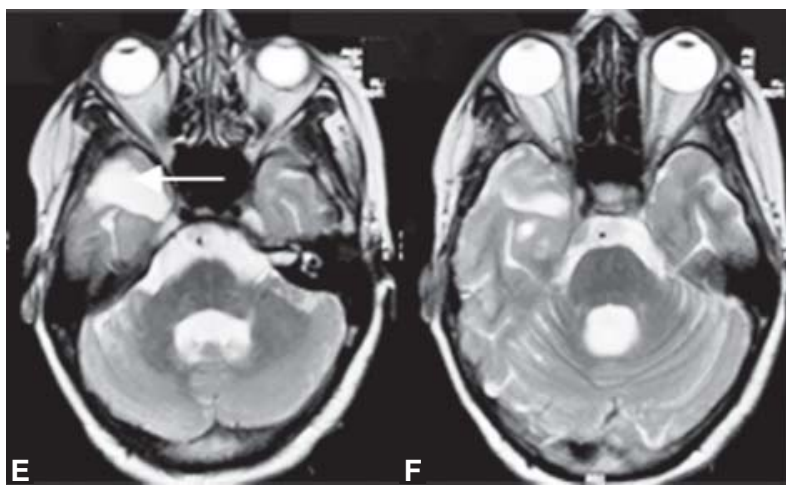

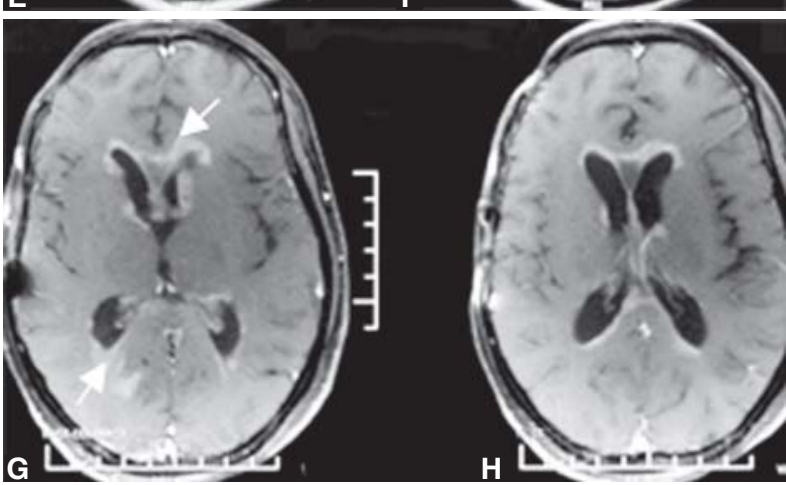

Figs 1A to H: (A) F-18 FDG PET image showing intense FDG uptake in the temporal pole on the right side extending along the medial temporal lobe, (B) F-18 FLT PET image showing increased tracer uptake in the right temporal pole at the site of recurrence, (C) F-18 FDG PET/CT image showing increased tracer uptake along the anterior horn of the lateral ventricle on the left side, (D) F-18 FLT PET/CT image demonstrating the uptake in the periventricular locations which are not seen on FDG PET image, ( $E$ and F) axial T2-weighted MRI sections at the level of temporal lobes showing hyperintense lesion on the right side corresponding to the high uptake on FDG PET, (G and $\mathrm{H})$ axial postcontrast T1-weighted MRI images showing periventricular-subependymal enhancing lesions also extending to corpus callosum

4. Chen W, Cloughesy T, Kamdar N, Satyamurthy N, Bergsneider M, Liau L, et al. Imaging proliferation in brain tumors with 18F-FLT PET: Comparison with 18F-FDG. J Nucl Med 2005;46:945-52.

5. Hatakeyama T, Kawai N, Nishiyama Y, Yamamoto Y, Sasakawa Y, Ichikawa T, et al. 11C-methionine (MET) and 18F-fluorothymidine (FLT) PET in patients with newly diagnosed glioma. Eur J Nucl Med Mol Imaging 2008;35: 2009-17.

6. Spence AM, Muzi M, Link JM, O’Sullivan F, Eary JF, Hoffman JM, et al. NCI-sponsored trial for the evaluation of safety and preliminary efficacy of 3'-deoxy-3'-[18F] fluorothymidine (FLT) as a marker of proliferation in patients with recurrent gliomas: Preliminary efficacy studies. Mol Imaging Biol 2009;11:343-55.

7. Price SJ, Fryer TD, Cleij MC, Dean AF, Joseph J, Salvador R, et al. Imaging regional variation of cellular proliferation in gliomas using 3'-deoxy-3'-[18F] fluorothymidine positronemission tomography: An image-guided biopsy study. Clin Radiol 2009;64:52-63.

8. Saga T, Kawashima H, Araki N, Takahashi JA, Nakashima Y, Higashi T, et al. Evaluation of primary brain tumors with FLT-PET: Usefulness and limitations. Clin Nucl Med 2006;31: 774-80.

9. Yamamoto Y, Wong TZ, Turkington TG, Hawk TC, Reardon DA, Coleman RE. 3'-deoxy-3'-[F-18]fluorothymidine positron emission tomography in patients with recurrent glioblastoma multiforme: Comparison with Gd-DTPA enhanced magnetic resonance imaging. Mol Imaging Biol 2006;8: 340-47.

\section{ABOUT THE AUTHORS}

\section{Raghava Kashyap}

Senior Resident, Department of Nuclear Medicine and PET Postgraduate Institute of Medical Education and Research Chandigarh, India

\section{Manoj Kumar Tiwari}

Professor, Department of Neurosurgery, Postgraduate Institute of Medical Education and Research, Chandigarh, India

\section{Raja Senthil}

Senior Resident, Department of Nuclear Medicine and PET Postgraduate Institute of Medical Education and Research Chandigarh, India

\section{Anish Bhattacharya}

Additional Professor, Department of Nuclear Medicine, Postgraduate Institute of Medical Education and Research, Chandigarh, India

\section{Vivek Gupta}

Associate Professor, Department of Radiodiagnosis, Postgraduate Institute of Medical Education and Research, Chandigarh, India

\section{Bhagwant Rai Mittal (Corresponding Author)}

Professor and Head, Department of Nuclear Medicine, Postgraduate Institute of Medical Education and Research, Chandigarh-160012, India Phone: 91-1722756722,Fax: 91-1722742858, e-mail:brmittal@yahoo.com 